\title{
Black-Box Circular-Secure Encryption beyond Affine Functions
}

\author{
Zvika Brakerski ${ }^{1}$, Shafi Goldwasser ${ }^{2}$, and Yael Tauman Kalai ${ }^{3}$ \\ 1 Weizmann Institute of Science \\ zvika.brakerski@weizmann.ac.il \\ 2 Weizmann Institute of Science and MIT \\ shafi@theory.csail.mit.edu \\ 3 Microsoft Research \\ yael@microsoft.com
}

\begin{abstract}
We show how to achieve public-key encryption schemes that can securely encrypt nonlinear functions of their own secret key. Specifically, we show that for any constant $d \in \mathbb{N}$, there exists a public-key encryption scheme that can securely encrypt any function $f$ of its own secret key, assuming $f$ can be expressed as a polynomial of total degree $d$. Such a scheme is said to be key-dependent message (KDM) secure w.r.t. degree- $d$ polynomials. We also show that for any constants $c, e$, there exists a public-key encryption scheme that is KDM secure w.r.t. all Turing machines with description size $c \log \lambda$ and running time $\lambda^{e}$, where $\lambda$ is the security parameter. The security of such public-key schemes can be based either on the standard decision Diffie-Hellman (DDH) assumption or on the learning with errors (LWE) assumption (with certain parameters settings).

In the case of functions that can be expressed as degree- $d$ polynomials, we show that the resulting schemes are also secure with respect to key cycles of any length. Specifically, for any polynomial number $n$ of key pairs, our schemes can securely encrypt a degree- $d$ polynomial whose variables are the collection of coordinates of all $n$ secret keys. Prior to this work, it was not known how to achieve this for nonlinear functions.

Our key idea is a general transformation that amplifies KDM security. The transformation takes an encryption scheme that is KDM secure w.r.t. some functions even when the secret keys are weak (i.e. chosen from an arbitrary distribution with entropy $k$ ), and outputs a scheme that is KDM secure w.r.t. a richer class of functions. The resulting scheme may no longer be secure with weak keys. Thus, in some sense, this transformation converts security with weak keys into amplified KDM security.
\end{abstract}

\section{Introduction}

Secure encryption is one of the most fundamental tasks in cryptography, and significant work has gone into defining and attaining it. In many classical notions of secure encryption, it is assumed that the plaintext messages to be encrypted are independent of the secret decryption keys. However, over the years, it was observed that in some situations the plaintext messages do depend on the secret keys. This more demanding setting, termed key-dependent message security 
(KDM security) by Black, Rogoway and Shrimpton [7, has received much attention in recent years [12, 1, 21, 19, 5, 4, 20, 8, 17, 11, 3, 6, 9].

KDM security w.r.t. a class $\mathcal{F}$ of efficiently computable functions is modeled as follow 1 . An adversary is given public keys $\mathrm{pk}_{1}, \ldots, \mathrm{pk}_{n}$ and can access an oracle $\mathcal{O}$ that upon receiving a query $(i, f)$, where $f$ is a function in the class $\mathcal{F}$, and $i \in[n]$ is an index, returns an encryption of $f\left(\mathrm{sk}_{1}, \ldots, \mathrm{sk}_{n}\right)$ under the public key $\mathrm{pk}_{i}$. The scheme is $\mathrm{KDM}^{(n)}$ secure w.r.t. $\mathcal{F}$, where $n$ is the number of public keys, if the adversary cannot distinguish between the oracle $\mathcal{O}$ and an oracle that always returns an encryption of (say) the all-zero string. In particular, in $\mathrm{KDM}^{(1)}$ security, the adversary is given a single public key pk and can ask for encryptions (under pk) of functions of the corresponding secret key sk.

Starting with the breakthrough work of Boneh, Halevi, Hamburg and Ostrovsky [8] and continuing with the work of Applebaum, Cash, Peikert and Sahai [3], it is known how to achieve KDM security under a variety of computational assumptions. However, the above works achieve security only w.r.t. affine functions of the secret key, leaving unanswered the question of achieving security w.r.t. richer classes of functions.

The motivation to explore beyond affine functions is a straightforward extension of that provided in [8]: Assume a secret key is stored on a hard drive which is being encrypted as a part of a backup process. The encrypted contents thus depend on the secret key in a way that may not necessarily be affine (conditioned on the file type and the file system used).

Heitner and Holenstein [17] gave impossibility results with regards to blackbox constructions of $\mathrm{KDM}^{(1)}$-secure encryption (even in the symmetric case). They showed that $\mathrm{KDM}^{(1)}$ security w.r.t. poly-wise independent functions is not black-box reducible to one-way trapdoor permutations, and also that $\mathrm{KDM}^{(1)}$ security w.r.t. all functions is not black-box reducible to essentially any cryptographic assumption.

In a work independent and concurrent to ours, Barak, Haitner, Hofheinz and Ishai [6] show how to overcome the latter black-box separation of [17]. They give a very strong positive result, showing that for any polynomial $p$ there exists a $\mathrm{KDM}^{(1)}$-secure schemes w.r.t. all functions computable by circuits of size at most $p$, based on either the DDH or LWE assumptions. They also achieve $\mathrm{KDM}^{(n)}$ security at the cost of having the ciphertext length depend on the number of users $n$. Altogether, our work and theirs are complementary and achieve incomparable results. See a detailed comparison at the end of Section 1.1 below.

\subsection{Our Results}

We provide a general transformation that amplifies KDM security. Throughout this work, we restrict our attention to public-key encryption schemes in which the keygeneration algorithm works by first sampling a secret key and then applying some, possibly randomized, function to produce the public key. Many known encryption

${ }^{1}$ We define KDM security in the public-key setting since this is the focus of this work.

A similar definition can be provided for the symmetric setting. 
schemes have this property, e.g. [26, 14, 24, 8, 3] and others. We say that an encryption scheme is entropy- $k$ KDM-secure if it is KDM-secure even when the secret key is sampled from an arbitrary distribution with min-entropy $k$, and the computation of the public key is performed with perfect randomnes:2. Our transformation starts with an encryption scheme $\mathcal{E}=(G, E, D)$ that is entropy- $k \mathrm{KDM}^{(n)}$-secure w.r.t. some class of functions $\mathcal{F}$, and converts it into another scheme $\mathcal{E}^{*}=\left(G^{*}, E^{*}, D^{*}\right)$, which is $\mathrm{KDM}^{(n)}$ secure w.r.t. a larger class of functions $\mathcal{F}^{\prime}$.

Theorem 1.1 (informal). Let $\mathcal{E}=(G, E, D)$ be a public-key encryption scheme that is entropy- $k \mathrm{KDM}^{(n)}$-secure w.r.t. a function class $\mathcal{F}$. Let $\mathcal{S}$ denote the space of the secret keys of $\mathcal{E}$, and let $\mathcal{K}$ be any set of size at least $2^{k}$. Then for every deterministic, efficiently computable and injective mapping $\alpha: \mathcal{K} \rightarrow \mathcal{S}$ there exists an encryption scheme $\mathcal{E}_{\alpha}^{*}=\left(G^{*}, E^{*}, D^{*}\right)$, whose secret key, $\mathrm{sk}^{*}$, is chosen at random from $\mathcal{K}$, such that $\mathcal{E}_{\alpha}^{*}$ is $\mathrm{KDM}^{(n)}$ secure w.r.t. the function class $\mathcal{F}^{\prime}=\mathcal{F} \circ \alpha=\left\{(f \circ \alpha)\left(\mathrm{sk}_{1}^{*}, \ldots, \mathrm{sk}_{n}^{*}\right)=f\left(\alpha\left(\mathrm{sk}_{1}^{*}\right), \ldots, \alpha\left(\mathrm{sk}_{n}^{*}\right)\right): f \in \mathcal{F}\right\}$.

For example, one can think of $\alpha(\mathrm{sk})$ as the vector of all monomials of degree $d$; namely, $\alpha\left(x_{1}, \ldots, x_{k}\right)=\left(\prod_{i \in I} x_{i}\right)_{|I| \leq d}$, where sk $=\left(x_{1}, \ldots, x_{k}\right) \in\{0,1\}^{k}$. Another example is where $\alpha(\mathrm{sk})$ is the vector of all Turing machines with description length $O(\log k)$ and running time at most $t$ (for some polynomial $t$ ), applied to sk. Namely, $\alpha(\mathrm{sk})=\langle M(\mathrm{sk})\rangle_{M}$, where $M$ is a Turing machine with description length $O(\log k)$ that runs for at most $t$ steps on sk.

In the first example, if $\mathcal{F}$ is the class of all linear functions, then $\mathcal{F}^{\prime}=\mathcal{F} \circ \alpha$ is the class of all degree $\leq d$ polynomials. In the the second example, if $\mathcal{F}$ contains the identity function, then $\mathcal{F}^{\prime}=\mathcal{F} \circ \alpha$ contains all the Turing machines with description length $O(\log k)$ and running time at most $t$.

We emphasize that in Theorem 1.1, we start with a scheme $\mathcal{E}$ that is entropy$k \mathrm{KDM}^{(n)}$-secure w.r.t. a function class $\mathcal{F}$, and end up with a scheme $\mathcal{E}_{\alpha}^{*}$ that is not necessarily entropy- $k$ secure anymore. However, it is $\mathrm{KDM}^{(n)}$-secure w.r.t. a (supposedly richer) function class $\mathcal{F}^{\prime}$. Therefore this theorem gives a way to convert security with weak keys, into enhanced KDM security, thus showing a formal connection between the two notions 3 . Another connection between these notions in the symmetric encryption case, was shown by Canetti et. al. 13, in the context of obfuscation of multi-bit point functions: Loosely speaking, they show that an encryption scheme that is entropy- $k$ KDM-secure implies a multi-bit point obfuscators, and vice versa. However, showing a direct implication between the notions (or showing that one does not exist) remains an interesting open problem.

We apply Theorem 1.1 to the schemes of [8] and [3] to obtain Theorems 1.2 and 1.3. respectively, presented below. In order to do that, we will argue that these schemes (or rather, a slight modification thereof) are entropy- $k \mathrm{KDM}^{(1)}$ secure. In what follows, $\lambda$ denotes the security parameter.

\footnotetext{
${ }^{2}$ This notion is different from security with key-leakage, where the leakage may depend on the public key.

${ }^{3}$ We stress that our reduction does not yield that leakage resiliency by itself implies KDM security; rather, we show that leakage resiliency on top of KDM security enables amplifying the KDM security property.
} 
Theorem 1.2 (informal). Assume the DDH assumption in a group $\mathbb{G}$ of order $q$, and let $g$ be any generator of $\mathbb{G}$. Then, for any class $\mathcal{H}=\left\{h_{1}, \ldots, h_{\ell}\right.$ : $\left.h_{i} \in\{0,1\}^{k} \rightarrow\{0,1\}\right\}$ of poly $(\lambda)$-time computable functions, with cardinality $\ell=\operatorname{poly}(\lambda)$, there exists a $\mathrm{KDM}^{(1)}$-secure encryption scheme w.r.t. the class of functions

$$
\mathcal{F}_{\mathcal{H}}=\left\{f\left(g^{\mathbf{x}}\right)=g^{\sum_{i \in[\ell]} t_{i} h_{i}(\mathbf{x})+w}: \mathbf{x} \in\{0,1\}^{k},(\mathbf{t}, w) \in \mathbb{Z}_{q}^{\ell} \times \mathbb{Z}_{q}\right\} .
$$

In this scheme, the secret key is a vector in $\mathbb{G}^{k}$ whose $i^{\text {th }}$ coordinate is $g^{x_{i}} \in$ $\{1, g\}$. Theorem 1.2 is obtained by applying Theorem 1.1 to the public-key encryption scheme of [8], which is KDM secure w.r.t. affine functions in the exponent, using the mapping $\alpha\left(g^{\mathbf{x}}\right)=\left(g^{h_{1}(\mathbf{x})}, \ldots, g^{h_{\ell}(\mathbf{x})}\right)$.

In particular, taking $\mathcal{H}$ to be the class of all degree- $d$ monomials, we show that for any constant $d \in \mathbb{N}$, there exists a public-key encryption scheme that is $\mathrm{KDM}^{(1)}$-secure w.r.t. all polynomials of total degree $d$ (in the exponent). This is because degree- $d$ polynomials over $k$ variables can be viewed as affine functions applied to the vector of degree- $d$ monomials. A different selection of $\mathcal{H}$ implies that for any polynomial $t$, there exists a public-key scheme that is $\mathrm{KDM}^{(1)}$-secure w.r.t. all Turing machines of description length bounded by $\log t$ and running time bounded by 4 .

Theorem 1.3 (informal). Under the LWE assumption with modulus $q=p^{2}$, for a prime $p$, for any class $\mathcal{H}=\left\{h_{1}, \ldots, h_{\ell}: h_{i} \in\{0,1\}^{k} \rightarrow\{0,1\}\right\}$ of $\operatorname{poly}(\lambda)$ time computable functions, with cardinality $\ell=\operatorname{poly}(\lambda)$, there exists a $\operatorname{KDM}^{(1)}$ secure encryption scheme w.r.t. the class of functions

$$
\mathcal{F}_{\mathcal{H}}=\left\{f(\mathbf{x})=\sum_{i \in[\ell]} t_{i} h_{i}(\mathbf{x})+w \quad(\bmod p):(\mathbf{t}, w) \in \mathbb{Z}_{p}^{\ell} \times \mathbb{Z}_{p}\right\} .
$$

The secret key space in this scheme is $\{0,1\}^{k}$. The result is obtained by applying Theorem 1.1 to (a variant of) the public-key encryption scheme of [3], which is KDM secure w.r.t. affine functions, using the mapping $\alpha(\mathbf{x})=\left(h_{1}(\mathbf{x}), \ldots, h_{\ell}(\mathbf{x})\right)$.

In a similar manner to the DDH based result, appropriate selections of $\mathcal{H}$ imply a $\mathrm{KDM}^{(1)}$-secure scheme w.r.t. all polynomials of total degree $d$ and a $\mathrm{KDM}^{(1)}$-secure scheme w.r.t. all Turing machines of description length bounded by $\log t$ and running time bounded by $t$, for $t=\operatorname{poly}(\lambda)$.

This ability, to tailor an encryption scheme to the required set of functions, can be useful when, as a part of a cryptographic protocol, encryptions of certain functions of the secret key need to be transmitted.

We are able to extend the above results, using additional techniques (Theorem 1.1 will not suffice), and show that for the case of degree- $d$ polynomials, both schemes obtained above are in fact $\mathrm{KDM}^{(n)}$-secure, based on their respective assumptions. These results are stated in the theorems below.

${ }^{4}$ Bear in mind that any uniform function can be represented by a Turing machine of constant description. This means that for any uniform function $f$ (computable in time $t$ ), our scheme becomes secure asymptotically with the security parameter. 
Theorem 1.4 (informal). Under the DDH assumption, for any constant $d \in$ $\mathbb{N}$, there exists a public-key encryption scheme that is $\mathrm{KDM}^{(n)}$-secure w.r.t. degree-d polynomials in the exponent, for any $n=\operatorname{poly}(\lambda)$.

Theorem 1.5 (informal). Under the LWE assumption, for any constant $d \in$ $\mathbb{N}$, there exists a public-key encryption scheme that is $\mathrm{KDM}^{(n)}$-secure w.r.t. degree-d polynomials, for any $n=\operatorname{poly}(\lambda)$.

Additional Uses for Our Amplification Theorem. While Theorem 1.1 is stated in terms of public-key encryption, it in fact also works, in a straightforward manner, for other primitives such as symmetric encryption or pseudo-random functions (under an appropriate adaptation of the definitions of KDM and entropy- $k$ security). In this paper, though, we focus on public-key encryption.

One could also consider applying the theorem to the OAEP (i.e. random oracle) based scheme of Backes, Dürmuth and Unruh [4]. However, in order to do that, entropy- $k$ secure one-way trapdoor functions are required. Such are currently not known to exist, to the best of our knowledge, and thus we do not elaborate on this scheme.

Comparison With [6]. As mentioned above, a recent independent work of [6] achieves KDM security for a very rich class of functions: the class of functions computable by circuits of polynomial size $p$ (the polynomial $p$ affects the parameters of the scheme as we explain below). Their main technique is a non black-box use of the functions in the class, resulting in the ciphertext's containing a garbled circuit corresponding to a size- $p$ circuit. Our implementation, in contrast, makes black-box use of the functions and does not require garbled circuits. The downside is that the size of the function class has to be limited (as demonstrated by the negative result of [17]). Another difference is that in the $\mathrm{KDM}^{(n)}$ scheme of [6], the ciphertext size depends on $n$, unlike our schemes.

We also note that while the [6] framework applies only for public-key encryption, ours can be applied to symmetric encryption as well as other primitives.

\subsection{Our Techniques}

Let us present the intuition behind the KDM amplification theorem (Theorem 1.1). Given an encryption scheme $\mathcal{E}$ that is entropy- $k \mathrm{KDM}^{(n)}$-secure w.r.t. a function class $\mathcal{F}$, we construct the encryption scheme $\mathcal{E}^{*}$ as follows: The key generation algorithm $G^{*}$, rather than choosing the secret key from $\mathcal{S}$, chooses sk $\stackrel{\$}{\leftarrow} \mathcal{K}$, and sets pk to be the public key corresponding to the secret key $\alpha(\mathrm{sk})$. As an example, one can think of $\mathcal{K}=\{0,1\}^{k}, \mathcal{S}=\{0,1\}^{\ell}$ where $\ell=\sum_{i=0}^{d}\left(\begin{array}{c}k \\ i\end{array}\right)$, and $\alpha(\mathrm{sk})$ is the vector of all monomials of degree $d$; namely, $\alpha\left(x_{1}, \ldots, x_{k}\right)=$ $\left(\prod_{i \in I} x_{i}\right)_{|I| \leq d}$, where sk $=\left(x_{1}, \ldots, x_{k}\right) \in\{0,1\}^{k}$. Another example is where $\mathcal{K}=\{0,1\}^{k}, \mathcal{S}=\{0,1\}^{\text {poly }(k)}$, and $\alpha(\mathrm{sk})$ as being the vector of all Turing machines with description length $O(\log k)$ and running time at most $t$ (for some polynomial $t$ ), applied to sk. Namely, $\alpha(\mathrm{sk})=\langle M(\mathrm{sk})\rangle_{M}$, where $M$ is a Turing machine with description length $O(\log k)$ that runs for at most $t$ steps on sk. 
The encryption algorithm $E^{*}$ is identical to $E$. The decryption algorithm $D^{*}$ takes the secret key sk, computes $\alpha$ (sk), and decrypts the ciphertext by applying the decryption algorithm $D$ with the secret key $\alpha(\mathrm{sk}) \sqrt{5}$.

We next exemplify why the scheme $\mathcal{E}^{*}$ has amplified KDM security. Assume, for example, that $\mathcal{E}$ was entropy- $k \mathrm{KDM}^{(1)}$ secure w.r.t. all affine functions. Consider, as in the example above, $\alpha(\mathrm{sk})$ that is the vector of all monomials of degree $d$. Then $\mathcal{E}^{*}$ is still secure, because it applies the scheme $\mathcal{E}$ with a weak secret key of min-entropy $k$. Moreover, the fact that $\mathcal{E}$ is entropy- $k \mathrm{KDM}^{(1)}$ secure w.r.t. all affine functions, implies that the scheme $\mathcal{E}^{*}$ is secure w.r.t. all affine functions of $\alpha(\mathrm{sk})$, i.e. all degree $d$ polynomials of sk. Similarly, if $\alpha(\mathrm{sk})$ is the vector of all Turing machines with description length $O(\log k)$ and with running time at most $t$, applied to sk, then $\mathcal{E}^{*}$ is $\mathrm{KDM}^{(1)}$ secure w.r.t. all functions computed by these Turing machines.

Thus, Theorem 1.1 provides us with a generic tool to amplify KDM security of schemes that are entropy- $k$ KDM-secure to begin with. However, the question that remains is: Do there exist entropy- $k$ KDM-secure schemes?

$\mathrm{KDM}^{(1)}$ Security. [8, 3] presented encryption schemes that are $\mathrm{KDM}^{(1)}$-secure w.r.t. some classes of functions. We argue that these schemes are in fact entropy$k \mathrm{KDM}^{(1)}$-secure (for some setting of parameters). This enables us to apply Theorem 1.1 and amplify $\mathrm{KDM}^{(1)}$ security "for free". Specifically, this implies $\mathrm{KDM}^{(1)}$-secure schemes w.r.t. degree- $d$ polynomials or bounded description and bounded running time Turing machines.

$\mathrm{KDM}^{(n)}$ security. Two problems arise when trying to utilize Theorem 1.1 to obtain $\mathrm{KDM}^{(n)}$ security. First, a direct application of Theorem 1.1 may not produce the strongest result. Consider, for example, the case of bounded degree polynomials. Even if we had a scheme that was entropy- $k \mathrm{KDM}^{(n)}$-secure w.r.t. affine functions, Theorem 1.1 would only imply a scheme that is $\mathrm{KDM}^{(n)}$-secure w.r.t. bounded-degree polynomials where each monomial only contains variables of the same secret key. Second, we are not able to show entropy- $k \mathrm{KDM}^{(n)}$ security for any scheme and therefore cannot satisfy the conditions of the theorem.

To obtain Theorems 1.4 and 1.5 . therefore, additional ideas are required. Rather than applying Theorem 1.1 directly for $\mathrm{KDM}^{(n)}$, we consider the schemes obtained by Theorems 1.2 and 1.3 for the specific case where $\mathcal{H}$ is the class of all degree- $d$ monomials. We then show that these schemes are not only $\mathrm{KDM}^{(1)}$ secure w.r.t. degree- $d$ polynomials, but are also $\mathrm{KDM}^{(n)}$-secure w.r.t. the same class. We emphasize that monomials can contain variables from all secret keys in the system. This part contains the bulk of technical difficulty of this work.

While the proof for each scheme requires special treatment, the crux of the idea in both cases is similar. We use the "linear" behavior exhibited by both underlying schemes (in the DDH-based scheme, linearity is in the exponent) which

\footnotetext{
${ }^{5}$ We must require that $\alpha$ is deterministic so that $\alpha(\mathrm{sk})$ evaluates to the same value at each invocation (and thus is consistent with pk). It is interesting to explore whether similar techniques can be used when $\alpha$ is a randomized mapping (and thus can even increase the entropy of $\alpha$ (sk) compared to sk).
} 
enables the following form of homomorphism: starting from a single public key, that corresponds to a secret key sk, it is possible to generate a public key that corresponds to a linearly-related secret key. This is done without knowing the original secret key sk, only the (linear) relation. We need to be careful in utilizing this property: as it turns out (and hinted by the intuition of Theorem 1.1 provided above), we need to apply this homomorphism on secret keys whose coordinates are low-degree monomials. Therefore we cannot use arbitrary linear transformations to "switch" between secret keys. We solve this problem by presenting a class of linear transformations that do preserve the structure of the input secret key.

\subsection{Other Related Works and Notions}

One can consider an "entropy- $k$ " variant for any security measure for publickey encryption, analogously to our definition of entropy- $k$ KDM security; i.e., requiring that the scheme remains secure, in the relative measure, even when the secret key is sampled from an arbitrary entropy- $k$ distribution. This notion is incomparable to that of key-leakage resilience, defined by Akavia, Goldwasser and Vaikuntanathan [2]. On the one hand, the notion of entropy- $k$ security is weaker since imperfect randomness is only used to generate the secret key, while the computation of the corresponding public key uses perfect randomness. On the other hand, key-leakage resilience is weaker since it requires security to hold, with high probability, over some family of distributions, whereas entropy- $k$ security requires security to hold for all high min-entropy distributions.

In this work, we restructure the secret key of a public-key encryption scheme in order to achieve additional properties. Previous works also used a key distribution other than the obvious one to obtain stronger results. In the KDM-secure scheme of [8], binary vectors in the exponent of a group generator are used as secret keys, instead of the more natural selection of vectors in $\mathbb{Z}_{q}$. This is done in order to achieve KDM security w.r.t. the desired function class. In [22], the secret key distribution of the [8] scheme is again modified, this time using vectors of higher dimension than required, thus achieving security against key-leakage. The KDM-secure public-key scheme of [3] is very similar to that of [24], with one of the changes being that the secret key distribution is selected from a narrow Gaussian rather than being uniform. This is done, again, in order for KDM security to apply w.r.t. the desired set of functions.

In a followup work, Brakerski and Goldwasser [9] present a KDM (and memory leakage resilient) secure scheme based on the quadratic residuosity assumption. They then use our techniques to amplify the KDM security of their scheme by showing that it is entropy- $k$ KDM secure.

\subsection{Paper Organization}

We provide notation and standard definitions in Section 2, new definitions and tools appear in Section [3. The KDM amplification theorem (Theorem 1.1) is formally restated and proven in Section 4, with examples of applying it to specific 
function classes. Due to space limitations, we omit the discussion of our DDH based solution and refer the reader to the full version [10] for the full details. Our LWE based construction appears in Section 5, where Theorems 1.3 and 1.5 are formally restated. Many proofs are omitted, see full version [10].

\section{Notation and Definitions}

We denote scalars in plain lowercase $(x \in\{0,1\})$, vectors in bold lowercase $\left(\mathbf{x} \in\{0,1\}^{k}\right)$ and matrices in bold uppercase $\left(\mathbf{X} \in\{0,1\}^{k \times k}\right)$. All vectors are column vectors by default. The $i^{\text {th }}$ coordinate of $\mathbf{x}$ is denoted $x_{i}$. For a set $I$, we use $\mathbf{x}=\left\langle x_{i}\right\rangle_{i \in I}$ to denote a vector that is indexed by elements in $I$.

Vectors in $\{0,1\}^{k}$ are treated both as elements in $\mathbb{Z}_{q}^{k}$ (for an appropriately defined $q \in \mathbb{N}$ ) and as elements in $\mathbb{Z}_{2}^{k}$. We use standard arithmetic notation for arithmetics over $\mathbb{Z}_{q}^{k}$ and use $\mathbf{x} \oplus \mathbf{y}$ to denote the addition in $\mathbb{Z}_{2}^{k}$ (i.e. bitwise XOR operation).

Let $X$ be a probability distribution. We write $x \stackrel{\$}{\leftarrow} X$ to indicate that $x$ is sampled from $X . X^{n}$ denotes the $n$-fold product distribution of $X$. The uniform distribution over a set $S$ is denoted $U(S)$ but we also denote $x \stackrel{\$}{\leftarrow} S$ to abbreviate $x \stackrel{\$}{\leftarrow} U(S)$. The min entropy of a random variable $X$ is denoted $\mathbf{H}_{\infty}(X)$. For any function $f, f(X)$ denotes the random variable (or corresponding distribution) obtained by sampling $x \stackrel{\$}{\leftarrow} X$ and outputting $f(x)$.

We write $\operatorname{negl}(n)$ to denote an arbitrary negligible function, i.e. one that vanishes faster than the inverse of any polynomial.

The statistical distance between two distributions $X, Y$ is denoted $\operatorname{SD}(X, Y)$. Two ensembles $\left\{X_{n}\right\}_{n},\left\{Y_{n}\right\}_{n}$ are statistically indistinguishable if $\operatorname{SD}\left(X_{n}, Y_{n}\right)=$ $\operatorname{negl}(n)$, and are computationally indistinguishable if for every poly $(n)$-time adversary $\mathcal{A}$ it holds that $\left|\operatorname{Pr}\left[\mathcal{A}\left(X_{n}\right)=1\right]-\operatorname{Pr}\left[\mathcal{A}\left(Y_{n}\right)=1\right]\right|=\operatorname{negl}(n)$.

Let $M$ be a deterministic Turing Machine. We use $|M|$ to denote the description length of $M$ and use execute $\left(M, 1^{t}, x\right)$ to denote the content of $M$ 's output tape after running on $x$ for $t$ computation steps. Clearly execute $\left(M, 1^{t}, x\right)$ is computable in time poly $(|M|, t)$.

\subsection{Learning with Errors (LWE)}

We use the decisional version of the LWE (24]) assumption. For any $m, n, q \in \mathbb{N}$ such that $q>2$, all functions of the security parameter $\lambda$, and any probability distribution $\chi$ on $\mathbb{Z}_{q}$, the $\mathrm{LWE}_{q, m, n, \chi}$ assumption is that the distributions $(\mathbf{A}, \mathbf{A} \mathbf{s}+\mathbf{x})$ and $(\mathbf{A}, \mathbf{u})$ are computationally indistinguishable, where $\mathbf{A} \stackrel{\$}{\leftarrow} \mathbb{Z}_{q}^{m \times n}$, $\mathbf{s} \stackrel{\$}{\leftarrow} \mathbb{Z}_{q}^{n}, \mathbf{x} \stackrel{\$}{\leftarrow} \chi^{m}, \mathbf{u} \stackrel{\$}{\leftarrow} \mathbb{Z}_{q}^{m}$.

We remark that the search version of the assumption, where the challenge is to find $\mathbf{s}$, is equivalent to the decisional version, for prime $q$, under poly $(q)$-time reductions. It is shown in [3] that this equivalence also holds for $q=p^{e}$, for integer constant $e$ and prime $p$, provided that $\chi$ is a distribution over $\mathbb{Z}_{q}$ that produces an element in $\left\{-\frac{p-1}{2}, \ldots, \frac{p-1}{2}\right\}$ with all but negligible probability. 
Worst-case to average-case reductions $([24,23])$ can be used to obtain a connection between LWE instances and worst case lattice problems, for some (Gaussian like) distribution $\chi$.

Noise Distributions. In our construction, we use distributions that are derived from Gaussians. For any $\sigma>0$, we denote $D_{\sigma}(x)=e^{-\pi(x / \sigma)^{2}} / \sigma$, the (scaled) density function of the one dimensional Gaussian distribution. For any $q \in \mathbb{N}$ and $\sigma>0$ we define $\bar{\Psi}_{\sigma}$ to be the distribution over $\mathbb{Z}_{q}$ obtained by sampling

$y \stackrel{\$}{\leftarrow} D_{\sigma}$ and outputting $\lfloor q \cdot y\rceil(\bmod q)$. We define $D_{\mathbb{Z}^{m}, \sigma}$ to be the distribution over all $\mathbf{x} \in \mathbb{Z}^{m}$ such that $\operatorname{Pr}[\mathbf{x}]$ is proportional to $\prod_{i \in[m]} D_{\sigma}\left(x_{i}\right)$. We note that this distribution is efficiently sampleable for any $\sigma>0$.

\subsection{KDM Security}

A public-key encryption scheme $\mathcal{E}=(G, E, D)$ is defined by its key generation, encryption and decryption algorithms. The key generation algorithm $G$ takes as input the unary vector $1^{\lambda}$, where $\lambda$ is called the security parameter of the scheme. All other parameters of the scheme are parameterized by $\lambda$. We let $\mathcal{S}=\left\{\mathcal{S}_{\lambda}\right\}$ denote the space of secret keys and $\mathcal{M}=\left\{\mathcal{M}_{\lambda}\right\}$ denote the message space of the encryption scheme. We refer the reader to [15] for a formal definition of encryption schemes and their security.

In the scenario of key-dependent messages, we wish to model the case where functions of the secret key can be encrypted, and require that the resulting ciphertexts are indistinguishable from encryptions of 0 . We want our definition to apply also for the case of "key cycles" where a function of one user's secret key is encrypted by another's public key and vice versa. The most inclusive definition, therefore, is parameterized by the number of users $n$ and allows encrypting a function of the entire vector of $n$ secret keys under any of the corresponding public keys (this is sometimes referred to as "clique security"). An additional parameter to be considered is the set of functions of the secret key that we allow to encrypt. We use the definition presented in [8].

Formally, let $\mathcal{E}=(G, E, D)$ be a public key encryption scheme, $n>0$ be an integer, $\mathcal{S}=\left\{\mathcal{S}_{\lambda}\right\}$ be the space of secret keys, and let $\mathcal{F}=\left\{\mathcal{F}_{\lambda}\right\}$ be a class of functions such that $\mathcal{F}_{\lambda} \subseteq \mathcal{S}_{\lambda}^{n} \rightarrow \mathcal{M}_{\lambda}$.

We define the $\operatorname{KDM}^{(n)}$ game, w.r.t. the function class $\mathcal{F}$, played between a challenger and an adversary $\mathcal{A}$, as follows.

- Initialize. The challenger selects $b \stackrel{\$}{\leftarrow}\{0,1\}$ and generates, for all $i \in[n]$, key pairs $\left(\mathrm{sk}_{i}, \mathrm{pk}_{i}\right) \stackrel{\$}{\leftarrow} G\left(1^{\lambda}\right)$. The challenger then sends $\left\{\mathrm{pk}_{i}\right\}_{i \in[n]}$ to $\mathcal{A}$.

- Query. The adversary makes queries of the form $(i, f) \in[n] \times \mathcal{F}_{\lambda}$. For each query, the challenger computes $y \leftarrow f\left(\mathrm{sk}_{1}, \ldots, \mathrm{sk}_{n}\right)$ and sends the following ciphertext to $\mathcal{A}$.

$$
c \leftarrow\left\{\begin{array}{l}
E_{\mathrm{pk}_{i}}(y) \text { if } b=0 \\
E_{\mathrm{pk}_{i}}(0) \text { if } b=1 .
\end{array}\right.
$$

- Finish. $\mathcal{A}$ outputs a guess $b^{\prime} \in\{0,1\}$. 
Adversary $\mathcal{A}$ wins the game if $b^{\prime}=b$. The scheme is secure if the advantage $\mathrm{KDM}^{(n)} \operatorname{Adv}[\mathcal{A}, \mathcal{E}](\lambda)=\left|\operatorname{Pr}\left[b^{\prime}=b\right]-1 / 2\right|$ of any polynomial time $\mathcal{A}$ is negligible.

We use $\mathrm{KDM}_{\mathcal{F}}^{(n)}$ to denote KDM security w.r.t. the function class $\mathcal{F}$.

\section{New Definitions and Tools}

\subsection{Projective Encryption Schemes and Weak Keys}

Projection. Throughout this paper, we only consider encryption schemes that have a projection between the secret and public key. Namely, the key generation can be described as first sampling the secret key from some set and then applying an efficiently computable projection function (which can be randomized) to generate the public key.

Definition 3.1 (projection). Let $\mathcal{E}=(G, E, D)$ be a public-key encryption scheme. $\mathcal{E}$ is projective if $G\left(1^{\lambda}\right)=(\mathrm{sk}, \mathrm{pk}=\operatorname{Proj}(\mathrm{sk}))$ where $\mathrm{sk} \stackrel{\leftrightarrow}{\leftarrow} \mathcal{S}$ and $\operatorname{Proj}(\cdot)$ is an efficiently computable (possibly randomized) function.

We remark that many known encryption schemes are indeed projective, e.g. [26, 14, 24, 8, 3] and others. We further remark that any secure scheme can be modified to be projective by using the randomness of the key generation as the secret key. However such transformation does not preserve KDM security and thus we will need to require projection explicitly.

Weak Keys and Entropy-k Security. We are also interested in a more specific case where a (projective) scheme remains secure even when the key generation is "improper": the secret key is sampled from an arbitrary distribution on $\mathcal{S}$ that has min-entropy $k$. The projection is then applied to the sampled value.

We can think of an "entropy- $k$ variant" of any security notion $\sigma$, we thus provide a general definition. In this work, however, we instantiate this definition with $\sigma$ being KDM security.

Definition 3.2 (entropy- $k$ security). Let $\mathcal{E}=(G, E, D)$ be a projective publickey encryption scheme and let $\sigma$ be some security notion. Consider a distribution ensemble $\mathcal{D}=\left\{\mathcal{D}_{\lambda}\right\}$ over $\mathcal{S}=\left\{\mathcal{S}_{\lambda}\right\}$. Let $G_{\mathcal{D}}$ denote the following key-generator: $G_{\mathcal{D}}\left(1^{\lambda}\right)=\left(\right.$ sk, Proj(sk)) where sk $\leftarrow \mathcal{D}_{\lambda}$.

Let $k: \mathbb{N} \rightarrow \mathbb{R}^{+}$be some function. $\mathcal{E}$ is entropy- $k \sigma$-secure if for any ensemble $\mathcal{D}$ with $\mathbf{H}_{\infty}\left(\mathcal{D}_{\lambda}\right) \geq k(\lambda)$ it holds that $\mathcal{E}_{\mathcal{D}}\left(G_{\mathcal{D}}, E, D\right)$ is $\sigma$-secure.

We stress that entropy- $k$ security, as defined above, is a notion incomparable to that of key-leakage resilience (as defined in [2, 22]). On the one hand, the notion of entropy- $k$ security is weaker since imperfect randomness is only used to generate the secret key, while the projection $\operatorname{Proj}(\cdot)$ uses perfect randomness to compute the corresponding public key. On the other hand, key-leakage resilience is weaker since it requires security to hold with high probability over some family of distributions, whereas entropy- $k$ security requires security to hold for all high min-entropy distributions. 


\subsection{Transformations on Expanded Secret Keys}

Let $q$ be some modulus. The set of affine functions modulo $q$ on $\mathbb{Z}_{q}^{k}$ is

$$
\mathcal{F}_{\text {aff }}=\left\{f_{\mathbf{t}, w}(\mathbf{x})=\mathbf{t}^{T} \mathbf{x}+w:(\mathbf{t}, w) \in \mathbb{Z}_{q}^{k} \times \mathbb{Z}_{q}\right\} .
$$

Degree- $d$ polynomials over $k$ variables can be viewed as affine functions applied to the vector of degree- $d$ monomials. While we consider polynomials over $\mathbb{Z}_{q}$, we only apply them to binary variables, $\mathbf{x} \in\{0,1\}^{k}$. We define a mapping $\gamma_{k, d}$ that maps $\mathbf{x} \in\{0,1\}^{k}$ into the vector containing all monomials of degree $d$ of the variables of $\mathbf{x}$.

Definition 3.3 (the vector of monomials $\gamma_{k, d}$ ). For all $k, d \in \mathbb{N}$ and $\mathbf{x} \in$ $\{0,1\}^{k}$, we define the vector of all degree-d monomials in $\mathbf{x}$ by

$$
\gamma_{k, d}(\mathbf{x})=\left\langle\prod_{j \in J} x_{j}\right\rangle_{\substack{J \subseteq[k] \\|\bar{J}| \leq d}} .
$$

In other words, letting $\nu_{k, d}=\sum_{j=0}^{d}\left(\begin{array}{l}k \\ j\end{array}\right)$ denote the number of such degree-d monomials, $\gamma_{k, d}:\{0,1\}^{k} \rightarrow\{0,1\}^{\nu_{k, d}}$ is a mapping between vectors. We denote its image by $\Gamma_{k, d}=\left\{\gamma_{k, d}(\mathbf{x}): \mathbf{x} \in\{0,1\}^{k}\right\}$.

It follows immediately from the definition that $\gamma_{k, d}$ is injective, since $\left(\gamma_{k, d}\right.$ $(\mathbf{x}))_{\{i\}}=x_{i}$, and thus that $\left|\Gamma_{k, d}\right|=2^{k}$.

Intuitively, in the context of KDM security amplification, $\mathbf{x}$ is our "real" secret key, whereas $\gamma_{k, d}(\mathbf{x})$, the expanded version of $\mathbf{x}$, is used as a "secret key" for a scheme that is KDM-secure w.r.t. affine functions. This results in a KDM-secure scheme w.r.t. degree- $d$ polynomials.

We denote the set of all degree-d polynomials over $\mathbb{Z}_{q}$ with binary variables $\mathbf{x} \in\{0,1\}^{k}$ by

$$
\mathcal{F}_{d}=\left\{f_{\mathbf{t}}(\mathbf{x})=\mathbf{t}^{T} \cdot \gamma_{k, d}(\mathbf{x}): \mathbf{t} \in \mathbb{Z}_{q}^{\ell}\right\} .
$$

Note that $\gamma_{k, d}(\mathbf{x})_{\emptyset}=1$, i.e. the vector of monomials contains the empty monomial that always evaluates to 1 . Therefore there is no need for an additional free term $w$ as in the definition of affine function:6.

The following lemma states that that given $\mathbf{y} \in\{0,1\}^{k}$, we can efficiently compute a matrix $\mathbf{T} \in \mathbb{Z}_{q}^{\ell \times \ell}$ such that for all $\mathbf{x} \in\{0,1\}^{k}$ it holds that $\mathbf{T}$. $\gamma_{k, d}(\mathbf{x})=\gamma_{k, d}(\mathbf{x} \oplus \mathbf{y})$. We think of $\mathbf{y}$ as the known relation between secret keys $\mathbf{x}$ and $\mathbf{x} \oplus \mathbf{y}$. The transformation $\mathbf{T}$ allows us to convert the expanded version of $\mathbf{x}$ to the expanded version of $\mathbf{x} \oplus \mathbf{y}$, i.e. to convert $\gamma_{k, d}(\mathbf{x})$ into $\gamma_{k, d}(\mathbf{x} \oplus \mathbf{y})$. For proof of the lemma, see the full version [10].

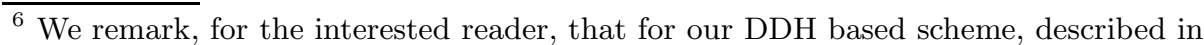
the full version [10, we need to define the analogous sets of affine functions in the exponent and degree-d polynomials in exponent over $\mathbb{G}^{k}$ :

$$
\begin{aligned}
\hat{\mathcal{F}}_{\text {aff }} & =\left\{h_{\mathbf{t}, w}\left(g^{\mathbf{x}}\right)=g^{\mathbf{t}^{T} \mathbf{x}+w}:(\mathbf{t}, w) \in \mathbb{Z}_{q}^{k} \times \mathbb{Z}_{q}\right\} \\
\hat{\mathcal{F}}_{d} & =\left\{h_{\mathbf{t}}\left(g^{\mathbf{x}}\right)=g^{\mathbf{t}^{T} \cdot \boldsymbol{\gamma}_{k, d}(\mathbf{x})}: \mathbf{t} \in \mathbb{Z}_{q}^{\ell}\right\},
\end{aligned}
$$

where $\mathbb{G}$ is a group of order $q$ and $g$ is a generator of $\mathbb{G}$. 
Lemma 3.4. For all $k, d, q \in \mathbb{N}$ such that $q>2$, there exists an efficiently computable function $\mathrm{T}_{k, d, q}:\{0,1\}^{k} \rightarrow \mathbb{Z}_{q}^{\ell \times \ell}$, where $\ell=\nu_{k, d}$, such that setting $\mathbf{T}=\mathbf{T}_{k, d, q}(\mathbf{y})$, for all $\mathbf{x} \in\{0,1\}^{k}$ it holds that $\mathbf{T} \cdot \gamma_{k, d}(\mathbf{x})=\gamma_{k, d}(\mathbf{x} \oplus \mathbf{y})$. Moreover $\mathbf{T}$ is an involution, i.e. $\mathbf{T}^{2}$ is the identity matrix.

\section{Amplification of KDM Security}

In this section we give a general result: We show that an entropy- $k$ KDM-secure scheme, w.r.t. a certain class of functions, can be converted into various schemes that are KDM-secure w.r.t. richer classes. We start by stating the general result in Section 4.1 and then, in Section 4.2, we present corollaries for specific classes of functions.

\subsection{Main Theorem}

Before stating our theorem, let us give some intuition for how KDM security can be amplified for projective entropy- $k$ schemes (as defined in Section 3.1).

Consider, for example, a projective encryption scheme $\mathcal{E}$ that is entropy- $k$ KDM-secure w.r.t. the class of indexing functions $\mathcal{I}=\left\{h_{i}(\mathbf{s})=s_{i}\right\}$ or, in other words, a bit by bit encryption of the secret key is secure. Entropy- $k$ security in particular means that we can sample the secret key sk $=\mathbf{s} \in\{0,1\}^{\ell}$ as follows: first, sample the first $k$ bits uniformly, call this part $\mathbf{x}$; then, set the remaining bits of $\mathbf{s}$ to $s_{i}=f_{i}(\mathbf{x})$, where $\left\{f_{i}\right\}_{i=k+1, \ldots, \ell}$ is an arbitrary class of efficiently computable deterministic functions. The resulting secret key distribution has min-entropy $k$ and thus $\mathcal{E}$ is still KDM-secure w.r.t. $\mathcal{I}$ with the resulting secret key distribution. Namely, $\mathcal{E}$ is secure w.r.t. the functions $h_{i}(\mathbf{s})=s_{i}=f_{i}(\mathbf{x})$. Therefore, we can convert $\mathcal{E}$ into a scheme $\mathcal{E}^{*}$ by setting the secret key in $\mathcal{E}^{*}$ to be $\mathbf{x}$. This $\mathcal{E}^{*}$ is KDM-secure w.r.t. indexing functions as well as the functions $\left\{f_{i}\right\}_{i=k+1, \ldots, \ell}$.

Theorem 1.1 (restated). Let $\mathcal{E}=(G, E, D)$ be a projective public-key encryption scheme that is entropy- $k \mathrm{KDM}^{(n)}$-secure w.r.t. a function class $\mathcal{F}$. Let $\mathcal{S}=\left\{\mathcal{S}_{\lambda}\right\}$ be the space of secret keys.

Let $\mathcal{K}=\left\{\mathcal{K}_{\lambda}\right\}$ be a family of sets such that $|\mathcal{K}| \geq 2^{k}$ and let $\alpha: \mathcal{K} \rightarrow \mathcal{S}$ be a deterministic, efficiently computable and injective function. Then there exists a projective encryption scheme $\mathcal{E}_{\alpha}^{*}=\left(G^{*}, E^{*}, D^{*}\right)$ with secret key space $\mathcal{K}$ that is $\mathrm{KDM}^{(n)}$ secure w.r.t. $\mathcal{F} \circ \alpha=\left\{(f \circ \alpha)\left(\mathrm{sk}_{1}, \ldots, \mathrm{sk}_{n}\right)=f\left(\alpha\left(\mathrm{sk}_{1}\right), \ldots, \alpha\left(\mathrm{sk}_{n}\right)\right)\right.$ : $f \in \mathcal{F}\}$.

Proof. Consider the ensemble $\mathcal{D}$ where $\mathcal{D}_{\lambda}=\alpha\left(U\left(\mathcal{K}_{\lambda}\right)\right)$ and consider the scheme $\mathcal{E}_{\mathcal{D}}=\left(G_{\mathcal{D}}, E, D\right)$ as in Definition 3.2, $\mathcal{E}_{\alpha}^{*}$ is similar to $\mathcal{E}_{\mathcal{D}}$ with the following modifications. $G^{*}\left(1^{\lambda}\right)$ first samples $\mathrm{sk}^{*} \stackrel{\$}{\leftarrow} \mathcal{K}$ and then computes pk $=\operatorname{Proj}^{*}\left(\mathrm{sk}^{*}\right)=$ $\operatorname{Proj}\left(\alpha\left(\mathrm{sk}^{*}\right)\right)$. Note that the distribution of the public keys is identical to that of $\mathcal{E}_{\mathcal{D}}$ while the distributions of secret keys differ. The encryption $E^{*}$ is performed identically to $E$. The decryption $D_{\mathrm{sk}^{*}}^{*}(c)$ is performed by first computing $\mathrm{sk}=\alpha\left(\mathrm{sk}^{*}\right)$ and then outputting $D_{\mathrm{sk}}(c)$. 
Since $\alpha$ is injective, it holds that $\mathbf{H}_{\infty}\left(\mathcal{D}_{\lambda}\right) \geq k$, and thus by definition, $\mathcal{E}_{\mathcal{D}}$ is $\mathrm{KDM}^{(n)}$-secure w.r.t. $\mathcal{F}$.

We next show that for any adversary $\mathcal{A}^{*}$ for the $\mathrm{KDM}^{(n)}$ game with $\mathcal{E}_{\alpha}^{*}$, there exists an adversary $\mathcal{A}$ for the $\mathrm{KDM}^{(n)}$ game with $\mathcal{E}_{\mathcal{D}}$ such that

$$
\operatorname{KDM}_{\mathcal{F}}^{(n)} \operatorname{Adv}\left[\mathcal{A}, \mathcal{E}_{\mathcal{D}}\right](\lambda)=\operatorname{KDM}_{\mathcal{F} \circ \alpha}^{(n)} \operatorname{Adv}\left[\mathcal{A}^{*}, \mathcal{E}_{\alpha}^{*}\right](\lambda),
$$

completing the proof of the theorem. Our adversary $\mathcal{A}$ will simulate $\mathcal{A}^{*}$ as follows.

- Initialize. Since the public key distributions of $\mathcal{E}_{\mathcal{D}}$ and $\mathcal{E}_{\alpha}^{*}$ are identical, $\mathcal{A}$ forwards its input $\mathrm{pk}_{1}, \ldots, \mathrm{pk}_{n}$ to $\mathcal{A}^{*}$.

- Queries. When $\mathcal{A}^{*}$ sends the query $(i, f \circ \alpha) \in[n] \times(\mathcal{F} \circ \alpha), \mathcal{A}$ sends the query $(i, f)^{7}$. Let $\mathrm{sk}_{i}^{*}$ denote the secret key corresponding to $\mathrm{pk}_{i}$ in $\mathcal{E}_{\alpha}^{*}$, then by definition $\mathrm{sk}_{i}=\alpha\left(\mathrm{sk}_{i}^{*}\right)$ is the secret key corresponding to $\mathrm{pk}_{i}$ in $\mathcal{E}_{\mathcal{D}}$. Therefore $f\left(\mathrm{sk}_{1}, \ldots, \mathrm{sk}_{n}\right)=(f \circ \alpha)\left(\mathrm{sk}_{1}^{*}, \ldots, \mathrm{sk}_{n}^{*}\right)$, and $\mathcal{A}$ can forward the answer to $\mathcal{A}^{*}$. Thus, $\mathcal{A}$ can simulate any query made by $\mathcal{A}^{*}$ during the game.

- Finish. When $\mathcal{A}^{*}$ returns $b^{\prime}, \mathcal{A}$ also terminates and returns the same $b^{\prime}$.

Since $\mathcal{A}$ simulates $\mathcal{A}^{*}$ exactly, it follows that $\mathcal{A}$ achieves the same advantage in the $\mathrm{KDM}^{(n)}$ game with $\mathcal{E}_{\mathcal{D}}$ as $\mathcal{A}^{*}$ does with $\mathcal{E}_{\alpha}^{*}$.

\subsection{Exemplifying for Specific Function Classes}

We demonstrate specific cases where Theorem 1.1 amplifies KDM security. We restrict our attention to $\mathrm{KDM}^{(1)}$ security (see discussion below).

- Bounded description functions. We first show how to amplify the class of indexing functions $\mathcal{I}=\left\{h_{i}(\mathbf{s})=s_{i}\right\}$ into the class of all functions computable by a Turing machine with bounded description length and bounded running time. Let $\mathcal{E}$ be an entropy- $k \mathrm{KDM}^{(1)}$-secure encryption scheme w.r.t. the class of indexing functions, with message space $\mathcal{M}=\{0,1\}$ and secret key space $\mathcal{S}=\{0,1\}^{\ell}$. Let $\mathcal{K}=\{0,1\}^{k}$ and $\alpha(\mathbf{x})=\left\langle\operatorname{execute}\left(M, 1^{t(\lambda)}, \mathbf{x}\right)\right\rangle_{|M| \leq \log \ell}$ where $t(\cdot)$ is some (fixed) polynomial. Then $\mathcal{E}_{\alpha}^{*}$, defined in the proof of Theorem 1.1 is $\mathrm{KDM}^{(1)}$-secure w.r.t. all functions computable by a Turing machine with description length $\log \ell$ and running time $t(\lambda) 8$.

- Bounded degree polynomials. We now show how to amplify the class of affine functions into the class of bounded degree polynomials. Let $\mathcal{E}$ be an entropy- $k$ $\mathrm{KDM}^{(1)}$-secure encryption scheme w.r.t. the class of affine functions $\mathbb{F}^{\ell} \rightarrow \mathbb{F}$, with $\mathcal{M}=\mathbb{F}$ and $\mathcal{S} \subseteq \mathbb{F}^{\ell}$, for a finite field $\mathbb{F}$. Let $\mathcal{K}=\{0,1\}^{k} \subseteq \mathbb{F}^{k}$ and let $d$ be such that $\ell=\nu_{k, d}$ (see Definition 3.3), this implies that $d$ is at least $\frac{\log \ell}{\log (k+1)}$. Consider $\alpha(\mathbf{x})=\gamma_{k, d}(\mathbf{x})$, i.e. $\alpha$ contains all degree $d$ monomials. Then $\mathcal{E}_{\alpha}^{*}$, defined in the proof of Theorem 1.1, is $\mathrm{KDM}^{(1)}$-secure w.r.t. all degree- $d$ polynomials $\mathbb{F}^{k} \rightarrow \mathbb{F}$.

${ }^{7}$ We represent $f \circ \alpha$ in such a way that enables to derive $f$.

${ }^{8}$ One has to be careful when showing that $\alpha$ is injective. We can either assume that the first $k$ coordinates of the output contain the input, or, if $\ell$ is sufficiently larger than $k$, we can rely on the short description and running time of the indexing functions. 
We provided examples only for the case of $\mathrm{KDM}^{(1)}$ security for two reasons. First, while in Section 5.2 we present entropy- $k \mathrm{KDM}^{(1)}$-secure schemes based on the LWE assumption 9 , we are unable to obtain entropy- $k \mathrm{KDM}^{(n)}$-secure schemes for $n>1$. Second, even if such exist, the result of applying Theorem 1.1 for the classes above would be weaker than expected. This is because while the functions in the class $\mathcal{F}$ are applied to the vector of $n$ secret keys, the mapping $\alpha$ is only applied to one secret key at a time. Therefore, the first example above would imply $\mathrm{KDM}^{(n)}$ security w.r.t. Turing machines that only take one of the secret keys as input; the second would imply $\mathrm{KDM}^{(n)}$ security w.r.t. degree- $d$ polynomials where each monomial only contains variables from one secret key.

\section{LWE Based KDM Security}

For any constant $d$, we present a scheme that is $\mathrm{KDM}^{(n)}$ secure w.r.t. all degree$d$ polynomials, $\mathcal{F}_{d}$. We also present a scheme that is $\mathrm{KDM}^{(1)}$-secure w.r.t. the class of all functions computed by Turing machines with description length at most $\log t$ and running time $t$, for some polynomial $t$ (more generally, w.r.t. any class of efficiently computable functions of polynomial cardinality). Our starting point is the LWE based scheme presented in [3], which we denote $\mathcal{E}_{\mathrm{ACPS}}$, which is extended using ideas from Section 4

First, in Section 5.1, we present the relevant previous work, in this case the scheme of [3], denoted $\mathcal{E}_{\mathrm{ACPS}}$. Then, in Section [5.2, we prove the entropy- $k$ $\mathrm{KDM}^{(1)}$ security of $\mathcal{E}_{\mathrm{ACPS}}$ w.r.t. affine functions $\mathcal{F}_{\text {aff }}$, and present the consequences of applying Theorem 1.1 to $\mathcal{E}_{\mathrm{ACPS}}$. Finally, in Section 5.3, we show that in the special case of degree- $d$ polynomials, we can in fact prove $\mathrm{KDM}^{(n)}$ security of the scheme obtained from Theorem 1.1 .

\subsection{Scheme $\mathcal{E}_{\text {ACPS }}$}

We present the $\mathcal{E}_{\text {ACPS }}[\mathcal{S}]$ scheme which is similar to the scheme presented in $[3]$. The only difference is that we take the distribution of secret keys as a parameter. We also use slightly different notation for consistency with the rest of this paper.

- Parameters. Let $p$ be a prime and $q=p^{2}$. We set $\ell, m \in \mathbb{N}$ to be polynomial functions of $\lambda$ such that $m \geq 2(\ell+1) \log q$. Let $\chi=\bar{\Psi}_{\sigma}$ for $\sigma=\sigma(\lambda) \in(0,1)$ such that $\sigma \leq \frac{1}{p \cdot \sqrt{m} \cdot \omega(\log \lambda)}$. We also fix some $\tau=\omega(\sqrt{\log \lambda})$. Finally, let $\mathcal{S} \subseteq \mathbb{Z}_{p}^{\ell}$. The secret key space is $\mathcal{S}$ and the message space is $\mathbb{Z}_{p}$.

- Key Generation. On input $1^{\lambda}$, sample $\mathbf{s} \stackrel{\$}{\leftarrow}$ and set sk $=\$ 10$. Then, sample $\mathbf{A} \stackrel{\$}{\leftarrow} \mathbb{Z}_{q}^{m \times \ell}$ and $\boldsymbol{\eta} \stackrel{\$}{\leftarrow} \chi^{m}$ and set pk $=(\mathbf{A}, \mathbf{A} \cdot \mathbf{s}+\boldsymbol{\eta}) \in \mathbb{Z}_{q}^{m \times \ell} \times \mathbb{Z}_{q}^{m}$.

- Encryption. Define the distribution $E_{\mathbf{A}, \mathbf{b}}$ in $\mathbb{Z}_{q}^{\ell} \times \mathbb{Z}_{q}$ as follows. $E_{\mathbf{A}, \mathbf{b}}$ samples $\mathbf{r} \stackrel{\$}{\leftarrow} D_{\mathbb{Z}^{m}, \tau}, e \stackrel{\$}{\leftarrow} \bar{\Psi}_{\tau^{\prime}}$ where $\tau^{\prime}=\tau \sqrt{m}\left(\sigma+\frac{1}{2 q}\right)$ and outputs $\left(\mathbf{A}^{T} \cdot \mathbf{r}, \mathbf{b}^{T} \cdot \mathbf{r}+e\right) \in \mathbb{Z}_{q}^{\ell} \times \mathbb{Z}_{q}$.

\footnotetext{
${ }^{9}$ An additional DDH based example is provided in the full version 10 .

${ }^{10}$ In [3, $\mathbf{s}$ is sampled from the distribution $\chi^{\ell}$.
} 
On input a public key pk $=(\mathbf{A}, \mathbf{b})$ and a message $w \in \mathbb{Z}_{p}$, the encryption algorithm samples $(\mathbf{u}, v) \stackrel{\$}{\leftarrow} E_{\mathbf{A}, \mathbf{b}}$ and outputs

$$
(\mathbf{u}, v+w \cdot p) .
$$

- Decryption. On input a secret key s and a ciphertext $(\mathbf{u}, c)$, the decryption algorithm outputs

$$
\left\lfloor\left(c-\mathbf{u}^{T} \cdot \mathbf{s} \quad(\bmod q)\right) / p\right\rceil \quad(\bmod p) .
$$

When $\sigma \leq \frac{1}{p \cdot \sqrt{m} \cdot \omega(\log \lambda)}$, correctness (for any $\mathbf{s} \in \mathbb{Z}_{p}^{\ell}$ ) follows directly from [3].

\subsection{Amplification of $\mathrm{KDM}^{(1)}$ Security}

We use Theorem 1.1 to amplify the $\mathrm{KDM}^{(1)}$ security of $\mathcal{E}_{\mathrm{ACPS}}$. We say that a finite set of functions, $\mathcal{H}=\left\{h_{1}, \ldots, h_{\ell}\right\}$, with a common domain, is entropy preserving if $\alpha_{\mathcal{H}}(x)=\left(h_{1}(x), \cdots h_{\ell}(x)\right)$ is an injective function.

Theorem 1.3 (restated). Let $p$ be a prime number that is super-polynomial in $\lambda$ and denote $q=p^{2}$. Let $m, \ell, \sigma, \chi$ be as in the parameters of $\mathcal{E}_{A C P S}$. Let $k \leq \ell$ and set $k^{\prime}=\frac{k-\omega(\log \lambda)}{\log q}$. Let $\beta=\beta(\lambda) \in(0,1)$ be such that $\frac{\beta}{\sigma}=\operatorname{negl}(\lambda)$ and denote $\chi^{\prime}=\bar{\Psi}_{\beta}$. Let $\mathcal{H}=\left\{h_{1}, \ldots, h_{\ell}: h_{i} \in\{0,1\}^{k} \rightarrow\{0,1\}\right\}$ be an entropy preserving class of efficiently computable functions with cardinality $\ell=\operatorname{poly}(\lambda)$. Then under the $\mathrm{LWE}_{q, m, k^{\prime}, \chi^{\prime}}$ assumption, there exists a public-key encryption scheme that is $\mathrm{KDM}^{(1)}$ secure w.r.t. function class

$$
\mathcal{F}_{\mathcal{H}}=\left\{f(\mathbf{x})=\sum_{i \in[\ell]} t_{i} h_{i}(\mathbf{x})+w \quad(\bmod p):(\mathbf{t}, w) \in \mathbb{Z}_{p}^{\ell} \times \mathbb{Z}_{p}\right\} .
$$

Before we outline the proof, let us discuss the parameters of our hardness assumption. The decisional $\mathrm{LWE}_{q, m, k^{\prime}, \chi^{\prime}}$ assumption (see Section 2.1) is equivalent to the search version under a poly $(q)$-time reduction. The search version, in turn, is shown in [24] to correspond to worst-case lattice problems, under quantum reductions. In 23, a classical reduction from other worst-case lattice problems to search LWE is shown. Thus, we can set $p$ and $q$ to be quasi-polynomial in $\lambda$, set $\beta \geq n / q$ and set $\frac{\sigma}{\beta}$ to be quasi-polynomial in $\lambda$ as well (recall that for correctness we must take $\sigma \leq \frac{1}{p \cdot \sqrt{m} \cdot \omega(\log \lambda)}$, so we cannot set $\sigma$ to be too large, but one can verify that a proper selection of parameters exists). Using such parameters we can relate the security of our scheme to either the worst case hardness of obtaining a quasi-polynomial approximation factor for a lattice problem such as GapSVP, using quasi-polynomial time quantum algorithms, or to the worst case hardness of obtaining a classical quasi-polynomial time algorithm for a lattice problem such as $\operatorname{GapSVP}_{\zeta, \gamma}$ with quasi-polynomial $\zeta$.

To prove Theorem [1.3. we employ Theorem 1.1. As a precondition, we will need to establish entropy- $k \mathrm{KDM}^{(1)}$ security for $\mathcal{E}_{\mathrm{ACPS}}$, which is not straightforward. We do this in two steps. First, we prove $\mathrm{KDM}^{(1)}$ security based on a 
nonstandard assumption. Then, we use a result of Goldwasser, Kalai, Peikert and Vaikuntanathan [16] that implies that for the parameters of Theorem 1.3. LWE reduces to our new assumption, thus ultimately basing our scheme on standard decisional LWE. We remark that it may be possible to achieve better parameters than stated in Theorem 1.3 using a more efficient reduction, if such exists. See full version [10] for details and proof.

In the specific case of using the set of all degree- $d$ monomials as the function class $\mathcal{H}$, we obtain a $\mathrm{KDM}^{(1)}$-secure scheme w.r.t. $\mathcal{F}_{d}$, all degree-d polynomials modulo $p$. We describe this scheme, $\mathcal{E}_{2} 11$, explicitly. In Section 5.3 we show that $\mathcal{E}_{2}$ is $\mathrm{KDM}^{(n)}$-secure w.r.t. $\mathcal{F}_{d} \cdot\left(\gamma_{k, d}, \nu_{k, d}, \Gamma_{k, d}\right.$ were defined in Definition 3.3.)

Encryption Scheme $\mathcal{E}_{2}$. Let $k, d \in \mathbb{N}$ and consider $p, q, m, \sigma, \chi, \tau$, as in the definition of $\mathcal{E}_{\mathrm{ACPS}}\left[\Gamma_{k, d}\right]$, specifically let $\ell=\nu_{k, d}$. The secret key space of $\mathcal{E}_{2}$ is $\{0,1\}^{k}$ and the message space is $\mathbb{Z}_{p}$.

- Key Generation. On input $1^{\lambda}$, select $\mathbf{x} \stackrel{\$}{\leftarrow}\{0,1\}^{k}$ and set sk $=\mathbf{x}$. We denote $\mathbf{s}=\gamma_{k, d}(\mathbf{x})$ and note that $\mathbf{s}$ is uniform in $\Gamma_{k, d}$. The public key pk is generated as in $\mathcal{E}_{\mathrm{ACPS}}\left[\Gamma_{k, d}\right]$. Namely, pk $=(\mathbf{A}, \mathbf{A} \cdot \mathbf{s}+\boldsymbol{\eta}) \in \mathbb{Z}_{q}^{m \times \ell} \times \mathbb{Z}_{q}^{m}$. Note that the distributions of the public keys in $\mathcal{E}_{2}$ and $\mathcal{E}_{\mathrm{ACPS}}\left[\Gamma_{k, d}\right]$ are identical.

- Encryption. On inputs a public key pk and message $w$, the encryption algorithm runs the encryption algorithm of $\mathcal{E}_{\mathrm{ACPS}}\left[\Gamma_{k, d}\right]$ with the same inputs.

- Decryption. On inputs a secret key sk $=\mathbf{x} \in\{0,1\}^{k}$ and a ciphertext $(\mathbf{u}, c)$, the decryption algorithm uses $\mathbf{x}$ to obtain $\mathbf{s}=\gamma_{k, d}(\mathbf{x})$. Decryption proceeds as in $\mathcal{E}_{\mathrm{ACPS}}\left[\Gamma_{k, d}\right]$, with inputs a secret key $\mathbf{s}$ and a ciphertext $(\mathbf{u}, c)$.

\section{3 $\mathrm{KDM}^{(n)}$ Security w.r.t. Degree-d Polynomials}

We show that $\mathcal{E}_{2}$ is $\mathrm{KDM}^{(n)}$-secure w.r.t. $\mathcal{F}_{d}$. For proof, see full version [10].

Theorem 1.5 (restated). Consider the scheme $\mathcal{E}_{2}$ with p being super-polynomial in $\lambda$. Let $k^{\prime}=\frac{k-\omega(\log \lambda)}{\log q}$ and let $\beta=\beta(\lambda) \in(0,1)$ be such that $\frac{\beta}{\sigma}=\operatorname{negl}(\lambda)$. Define $\chi^{\prime}=\bar{\Psi}_{\beta}$. Under the $\mathrm{LWE}_{q, m \cdot n, k^{\prime}, \chi^{\prime}}$ assumption, $\mathcal{E}_{2}$ is $\mathrm{KDM}^{(n)}$-secure w.r.t. the class of degree-d polynomials modulo $p$.

Note that if $\mathrm{LWE}_{q, m \cdot n, k^{\prime}, \chi^{\prime}}$ is hard for all $n=\operatorname{poly}(\lambda)$, then $\mathcal{E}_{2}$ is $\mathrm{KDM}^{(n)}$-secure for any polynomial number of "users". We also note that as in Theorem 1.3. the LWE assumption we rely on is related to worst-case lattice problems. See discussion in Section 5.2 for more details.

\section{References}

[1] Adão, P., Bana, G., Herzog, J., Scedrov, A.: Soundness of formal encryption in the presence of key-cycles. In: di Vimercati, S.D.C., Syverson, P.F., Gollmann, D. (eds.) ESORICS 2005. LNCS, vol. 3679, pp. 374-396. Springer, Heidelberg (2005)

$\overline{11}$ This scheme is denoted $\mathcal{E}_{2}$ for consistency with the full version [10] where $\mathcal{E}_{1}$ denotes our DDH based scheme. 
[2] Akavia, A., Goldwasser, S., Vaikuntanathan, V.: Simultaneous hardcore bits and cryptography against memory attacks. In: Reingold [25], pp. 474-495

[3] Applebaum, B., Cash, D., Peikert, C., Sahai, A.: Fast cryptographic primitives and circular-secure encryption based on hard learning problems. In: Halevi [18], pp. 595-618

[4] Backes, M., Dürmuth, M., Unruh, D.: OAEP is secure under key-dependent messages. In: Pieprzyk, J. (ed.) ASIACRYPT 2008. LNCS, vol. 5350, pp. 506-523. Springer, Heidelberg (2008)

[5] Backes, M., Pfitzmann, B., Scedrov, A.: Key-dependent message security under active attacks - brsim/uc-soundness of symbolic encryption with key cycles. In: CSF, pp. 112-124. IEEE Computer Society, Los Alamitos (2007)

[6] Barak, B., Haitner, I., Hofheinz, D., Ishai, Y.: Bounded key-dependent message security. In: Gilbert, H. (ed.) EUROCRYPT 2010. LNCS, vol. 6110, pp. 423-444. Springer, Heidelberg (2010)

[7] Black, J., Rogaway, P., Shrimpton, T.: Encryption-scheme security in the presence of key-dependent messages. In: Nyberg, K., Heys, H.M. (eds.) SAC 2002. LNCS, vol. 2595, pp. 62-75. Springer, Heidelberg (2003)

[8] Boneh, D., Halevi, S., Hamburg, M., Ostrovsky, R.: Circular-secure encryption from decision diffie-hellman. In: Wagner, D. (ed.) CRYPTO 2008. LNCS, vol. 5157, pp. 108-125. Springer, Heidelberg (2008)

[9] Brakerski, Z., Goldwasser, S.: Circular and leakage resilient public-key encryption under subgroup indistinguishability - (or: Quadratic residuosity strikes back). In: Rabin, T. (ed.) CRYPTO 2010. LNCS, vol. 6223, pp. 1-20. Springer, Heidelberg (2010)

[10] Brakerski, Z., Goldwasser, S., Kalai, Y.: Black-box circular-secure encryption beyond affine functions (full version of this paper). Cryptology ePrint Archive, Report 2009/485 (2009), http://eprint.iacr.org/

[11] Camenisch, J., Chandran, N., Shoup, V.: A public key encryption scheme secure against key dependent chosen plaintext and adaptive chosen ciphertext attacks. In: Joux, A. (ed.) EUROCRYPT 2009. LNCS, vol. 5479, pp. 351-368. Springer, Heidelberg (2009)

[12] Camenisch, J.L., Lysyanskaya, A.: An efficient system for non-transferable anonymous credentials with optional anonymity revocation. In: Pfitzmann, B. (ed.) EUROCRYPT 2001. LNCS, vol. 2045, pp. 93-118. Springer, Heidelberg (2001)

[13] Canetti, R., Tauman Kalai, Y., Varia, M., Wichs, D.: On symmetric encryption and point obfuscation. In: Micciancio, D. (ed.) TCC 2010. LNCS, vol. 5978, pp. 52-71. Springer, Heidelberg (2010)

[14] El Gamal, T.: A public key cryptosystem and a signature scheme based on discrete logarithms. In: Blakely, G.R., Chaum, D. (eds.) CRYPTO 1984. LNCS, vol. 196, pp. 10-18. Springer, Heidelberg (1985)

[15] Goldreich, O.: Foundations of Cryptography - Basic Applications. Cambridge University Press, Cambridge (2004)

[16] Goldwasser, S., Kalai, Y., Peikert, C., Vaikuntanathan, V.: Robustness of the learning with errors assumption (2009) (manuscript)

[17] Haitner, I., Holenstein, T.: On the (im)possibility of key dependent encryption. In: Reingold [25], pp. 202-219

[18] Halevi, S. (ed.): CRYPTO 2009. LNCS, vol. 5677. Springer, Heidelberg (2009)

[19] Halevi, S., Krawczyk, H.: Security under key-dependent inputs. In: Ning, P., di Vimercati, S.D.C., Syverson, P.F. (eds.) ACM Conference on Computer and Communications Security, pp. 466-475. ACM, New York (2007) 
[20] Hofheinz, D., Unruh, D.: Towards key-dependent message security in the standard model. In: Smart, N.P. (ed.) EUROCRYPT 2008. LNCS, vol. 4965, pp. 108-126. Springer, Heidelberg (2008)

[21] Laud, P., Corin, R.: Sound computational interpretation of formal encryption with composed keys. In: Lim, J.-I., Lee, D.-H. (eds.) ICISC 2003. LNCS, vol. 2971, pp. 55-66. Springer, Heidelberg (2004)

[22] Naor, M., Segev, G.: Public-key cryptosystems resilient to key leakage. In: Halevi [18], pp. $18-35$

[23] Peikert, C.: Public-key cryptosystems from the worst-case shortest vector problem: extended abstract. In: Mitzenmacher, M. (ed.) STOC, pp. 333-342. ACM, New York (2009)

[24] Regev, O.: On lattices, learning with errors, random linear codes, and cryptography. In: Gabow, H.N., Fagin, R. (eds.) STOC, pp. 84-93. ACM, New York (2005)

[25] Reingold, O. (ed.): TCC 2009. LNCS, vol. 5444. Springer, Heidelberg (2009)

[26] Rivest, R.L., Shamir, A., Adleman, L.M.: A method for obtaining digital signatures and public-key cryptosystems. Commun. ACM 21(2), 120-126 (1978) 